\title{
Long-Term Outcomes of Coronary Artery Bypass Grafting in Veterans with Ischemic Cardiomyopathy
}

\author{
Michael A. Napolitano, MD, ${ }^{1,2}$ K. Benjamin Lee, MD, ${ }^{1,2}$ Ethan S. Rosenfeld, MD, ${ }^{1,2}$ Sheena W. Chen, MD, ${ }^{1,2}$ \\ Andrew D. Sparks, MS, ${ }^{2}$ Christian D. Nagy, MD,${ }^{1}$ Michael D. Greenberg, MD, ${ }^{1}$ Gregory D. Trachiotis, MD $^{1,2}$ \\ ${ }^{1}$ Division of Cardiothoracic Surgery and Heart Center, Washington DC Veterans Affairs Medical Center, Washington, DC; \\ ${ }^{2}$ Department of Surgery, George Washington University, Washington, DC; ${ }^{3}$ Division of Cardiology and Heart Center, Washington \\ DC Veterans Affairs Medical Center, Washington, DC
}

\section{ABSTRACT}

Background: The Surgical Treatment for Ischemic Heart Failure (STICH) trial showed that surgical revascularization in ischemic cardiomyopathy (ICM) patients improves longterm mortality compared with medical treatment alone. This study examines how veterans with ICM undergoing revascularization fare against patients without ICM; it also examines the outcomes in the veteran population.

Methods: This is a retrospective review of a singlecenter database. From 2000 to 2018, 1,461 patients underwent isolated coronary artery bypass grafting (CABG). Two-hundred-one patients with an ejection fraction less than $35 \%$ were classified as the ICM cohort. The primary outcome was mortality. Secondary outcomes included postoperative complications. Subgroup analysis was performed within the ICM cohort comparing off-pump CABG (OPCAB) versus on-pump CABG (ONCAB).

Results: ICM patients had a higher incidence of myocardial infarction (MI), diabetes, chronic kidney disease (CKD), and preoperative intra-aortic balloon pump (IABP) use. The non-ICM cohort was more functionally independent. OPCAB was performed in $80.1 \%$ of ICM and $66.3 \%$ of non-ICM cohorts. There was no statistical difference between ICM and non-ICM cohorts in 30-day mortality (OR 1.94[0.79 - 4.75], $P=.15$ ). The ICM cohort had an increased 5-year mortality (OR 1.75[1.14 - 2.69], $P=.01$ ) and 10-year mortality (OR 1.71[1.09-2.67], $P=.02)$. The ICM cohort showed improved, although not statistically significant, short-term mortality with OPCAB compared with ONCAB (3.1\% versus $12.5 \%$, OR 0.31[0.05 - 1.82], $P=.20)$

Conclusion: Veterans with ICM undergoing CABG demonstrated similar short-term survival compared with nonICM veterans. The long-term survival in the ICM cohort still is inferior to patients without ICM. There is a trend toward improved short-term survival in patients with ICM undergoing OPCAB.

Received February 5, 2020; accepted April 8, 2020.

Correspondence: Gregory D. Trachiotis, MD, 50 Irving St. NW, Suite 2A-163, Washington, DC, 20422; 202-745-8590; (e-mail: Gregory.Trachiotis@va.gov).

\section{INTRODUCTION}

Cardiovascular disease (CVD) continues to be the leading cause of death in the United States. Heart failure (HF) contributes to $9.3 \%$ of those deaths [Benjamin 2018]. The prevalence of $\mathrm{HF}$ in the US is rising, with a current estimate of 6.2 million Americans afflicted with the disease compared with 5.7 million in 2012 [Benjamin 2018]. Despite advances in medical therapy, HF prevalence is projected to increase to more than 8 million people by 2030; therefore more effort and research needs to be devoted to HF prevention and optimizing treatment [Benjamin 2018].

While medical therapy for ischemic cardiomyopathy (ICM) has made significant advances over the past few decades, surgical techniques and equipment have improved as well [Rezapour 2018]. A combination of medical therapy plus surgical intervention has had optimal results. The Surgical Treatment for Ischemic Heart Failure Extension Study (STICHES) trial established that coronary artery bypass grafting $(\mathrm{CABG})$ is a promising treatment modality in patients with ICM [Velazquez 2016]. Long-term follow up of patients over 10 years in the STICHES trial further solidified the superior outcomes in the surgery group, particularly with regards to reduced mortality. Compared to medical therapy alone, the absolute risk reduction was $7 \%$ in patients who had a CABG along with medical therapy [Velazquez 2016].

While the STICHES trial has helped guide treatment algorithms for the general population with ICM, the outcomes of patients with ICM in the veteran population are not well established. The veteran population does not follow the same disease pattern as that of the general population. US veterans are at a significantly higher risk of developing CVD when compared with the standard US citizen [Assari 2014]. Assari et al found that veterans had a higher incidence of cardiovascular problems independent of socioeconomic disparity and comorbidities [Assari 2014]. This increased risk was thought to be owed in part to higher prevalence of comorbid psychiatric illness in the veteran population, such as posttraumatic stress disorder (PTSD).

With these broader differences in mind, we sought to investigate veterans with heart disease and their ability tolerate cardiac surgery. Approximately 350,000 US veterans suffer from HF, causing an annual mortality of $14.5 \%$ [Groeneveld 2018]. When evaluating surgical outcomes in 
Table 1. Patient demographics

\begin{tabular}{|c|c|c|c|}
\hline Age & $64.6+/-9.6$ & $65.0+/-9.0$ & .59 \\
\hline Race - African American & $45(22.4)$ & $282(22.4)$ & .95 \\
\hline Race - White & $76(37.8)$ & $506(40.2)$ & .95 \\
\hline Prior MI & $152(75.6)$ & $632(50.16)$ & $<.01$ \\
\hline Cerebrovascular disease & $41(20.4)$ & $265(21.0)$ & .84 \\
\hline Peripheral vascular disease & $60(30.0)$ & $307(24.4)$ & .09 \\
\hline Hyperlipidemia & $81(40.3)$ & $535(42.5)$ & .56 \\
\hline Hypertension & $191(95.0)$ & $1210(96.0)$ & .5 \\
\hline Chronic kidney disease & $85(42.3)$ & $397(31.5)$ & $<.01$ \\
\hline Prior $\mathrm{PCl}$ & $31(22.3)$ & $238(25.0)$ & .49 \\
\hline \multicolumn{4}{|l|}{ ASA Classification } \\
\hline III & $25(12.4)$ & $243(19.3)$ & .01 \\
\hline IV & $175(87.1)$ & $1016(80.6)$ & \\
\hline Preoperative IABP use & $93(46.3)$ & $70(5.6)$ & $<.01$ \\
\hline \multicolumn{4}{|l|}{ Surgical priority } \\
\hline Elective & $184(91.5)$ & $1172(93.0)$ & .75 \\
\hline
\end{tabular}

veterans affairs (VA) patients, one meta-analysis by Matula et al showed varying results with regards to morbidity and mortality in VA patients compared with private patients, depending on the surgical field [Matula 2010]. Specific to cardiac surgery, Rosenthal et al in a retrospective review discovered an increased mortality risk in patients undergoing CABG at a VA center compared with private sector hospitals [Rosenthal 2003]. Knowing these notable differences in civilian versus veteran patients, the objective of this study is to assess the outcomes of isolated CABG in veterans with ICM and examine whether the survival benefits of surgical revascularization for ischemic cardiomyopathy, as shown in the civilian data, translate to the veteran population.

\section{MATERIALS AND METHODS}

This is a retrospective review of a prospectively collected database from a single institution. Due to the retrospective nature of the study, Institutional Review Board approval was waived. All patients who underwent isolated CABG for all indications and level of operative priority from 2000-2018 were included in the study. The age range was $36-90$ years, with no age restriction or exclusion criteria. Patients were stratified into two cohorts for purposes of comparison: those with ICM and those without. ICM was defined as patients with coronary disease and an ejection fraction $<35 \%$. A subgroup analysis was performed in patients with ICM, who were further substratified based on whether cardiopulmonary bypass (CPB) was used (off-pump CABG (OPCAB) versus on-pump CABG $(\mathrm{ONCAB})$. Chi-square or Fisher exact test were used for categorical variables and independent t-test or Kruskal-Wallis test for continuous variables. Clinically relevant preoperative and operative characteristics with $P<.2$ in univariate between group comparisons were considered possible confounding variables and adjusted for in multivariate logistic regression modeling. Cumulative mortality events were calculated using Kaplan-Meier survival analysis with time-to-event and censoring calculated from the date of the surgery to the date of last encounter or death. The primary outcome of interest was mortality (30-day, 5-year, and 10-year), and secondary outcomes were cardiovascular, pulmonary, and renal composite morbidity outcomes, as well as unplanned reoperation and wound complications. Descriptions of these composite 
Table 1. Patient demographics [cont.]

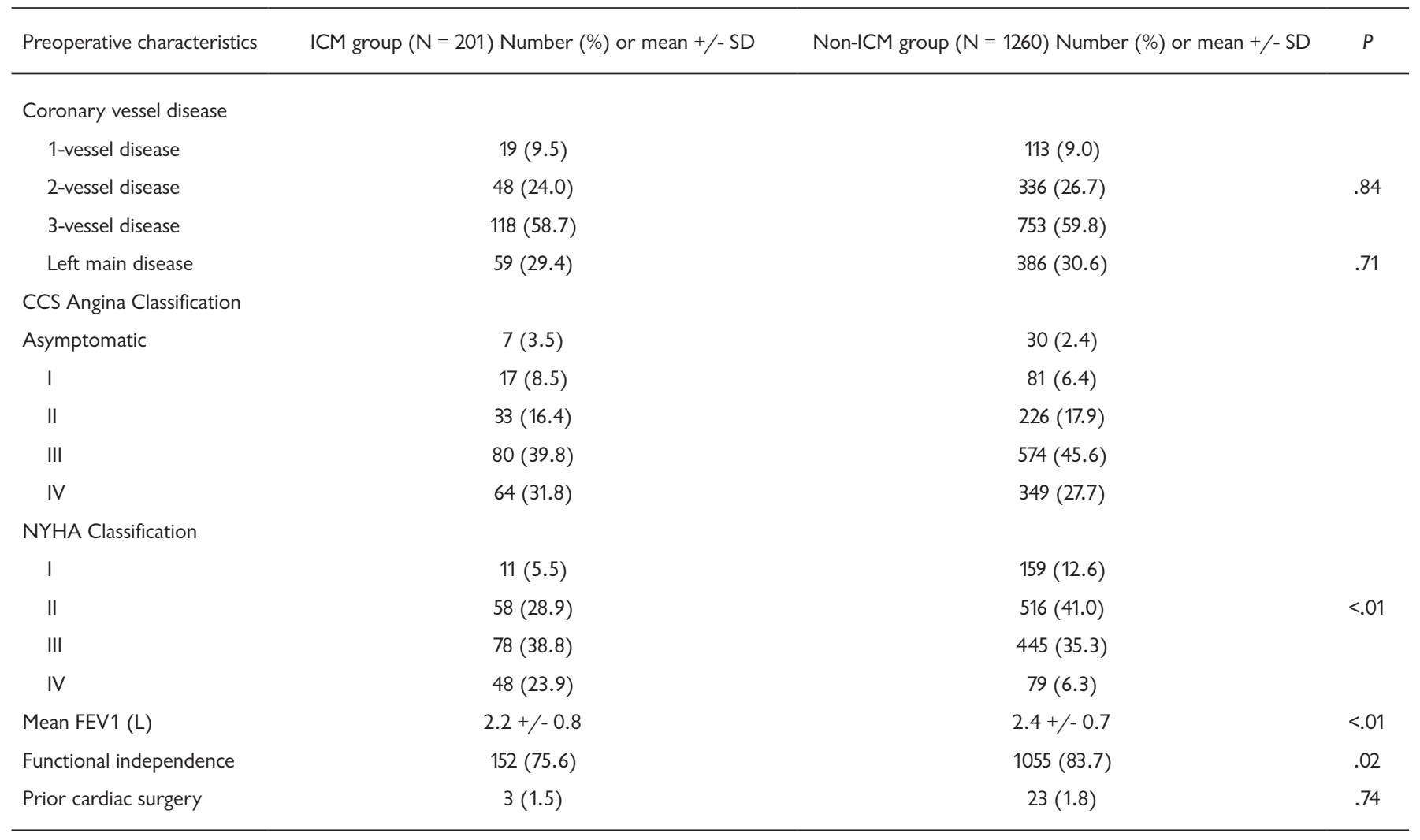

Abbreviations: ICM, ischemic cardiomyopathy; IABP, intra-aortic balloon pump; FEV1, forced expiratory volume in one second

outcomes are found in Table 2. All statistical analysis was performed using JMP Pro14 (SAS Institute, Cary, NC).

\section{RESULTS}

CABG was performed in 1,461 consecutive patients, of which 201 patients had ICM. The mean follow up was 9.9 years. The vast majority of patients were male $(99.0 \%)$, and there was no statistical difference in gender between ICM and non-ICM patients $(P=.42)$. Patients in the ICM group were more likely to have a history of diabetes $(P<.01)$, myocardial infarction $(\mathrm{MI})(P<.01)$, chronic kidney disease $(\mathrm{CKD})$ $(P<.01)$, higher New York Heart Association (NYHA) classification $(P=.01)$, and intra-aortic balloon pump (IABP) use $(P<.01)$. OPCAB was performed in $80.1 \%$ of ICM group and $66.3 \%$ of the non-ICM group $(P<0.01)$ (Table 1$)$.

Table 2 outlines the postoperative risk-adjusted outcomes. (Table 2) Our data did not show a statistically significant difference on multivariate analysis in 30-day mortality between patients with and without ICM (5.0\% versus $2.1 \%, P=.15)$. However, in the 10 -year follow up, mortality significantly was higher for the ICM group $(54.1 \%$ versus $34.9 \%$; aOR 1.71; Log-rank $P<.001)$. ICM group also had higher rates of pulmonary complications $(12.4 \%$ versus $5.2 \%, P<.01)$, which included ventilator $>48$ hours, reintubation events, tracheostomy, and pneumonia. The ICM group also had a higher rate of return to the operating room $(\mathrm{OR})$ for bleeding (3.5\% versus $1.2 \%, P=.02)$.

In the secondary analysis of OPCAB versus ONCAB in patients with ICM, there was no statistically significant difference in mortality over 10 years $(53.8 \%$ versus $55.2 \%$; Log-rank $P=.7019$, Table 1). Patients with ICM undergoing OPCAB had less pulmonary complications $(9.3 \%$ versus $25.0 \%, P<.01)$. The OPCAB group also had a lower incidence of return to the operating room for bleeding $(.6 \%$ versus $15 \%, P<.01)$.

\section{DISCUSSION}

Our data present a valuable insight into long-term outcomes of US veterans undergoing CABG for ICM, with results that mirror those seen in the civilian population. Our cohort showed a significant difference in 10-year mortality, with ICM patients having worse outcomes than their nonICM counterparts (Figure 1). This is not surprising given that patients suffering from ICM generally are sicker, often have a history of MI, have a decreased physiologic reserve, and have other comorbidities that lead to their demise, as seen in our study sample [Trachiotis 1998]. Overall, however, our 10-year mortality of ICM patients who had CABG was at 
$54.1 \%$, which is lower than the civilian data in the STICHES trial, which had their 10-year mortality at 58.9\% [Velazquez
[Puskas 2009]. Dewey et al also demonstrated that patients with reduced ventricular function fared better during CABG
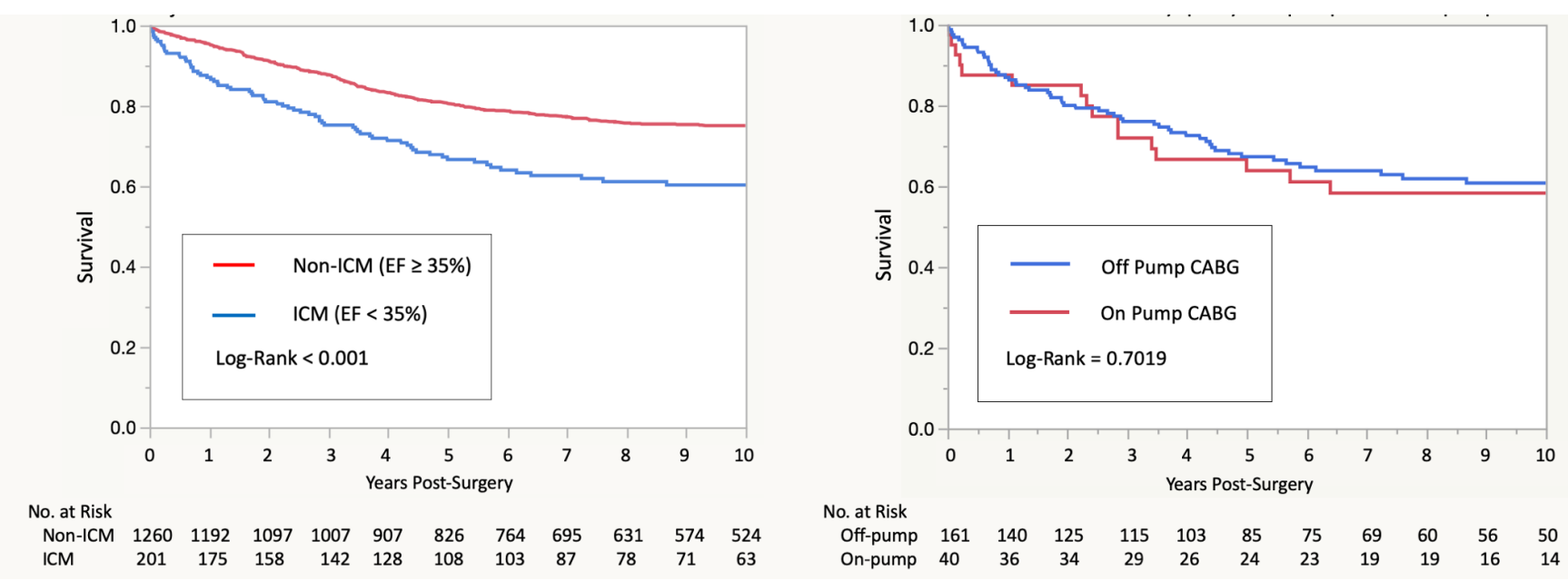

A) Survival after coronary artery bypass grafting in patients with ischemic cardiomyopathy versus patients with preserved ejection fraction; B) Survival after coronary artery bypass grafting in patients with ischemic cardiomyopathy: Off-pump versus on-pump.

2016]. Perhaps more importantly, the 30-day outcomes after CABG did not significantly differ between ICM and nonICM patients, which suggests that HF patients can tolerate the physiologic stressors of CABG and should thus be considered for this operation, as the STICHES trial has shown it to be beneficial for that patient population [Velazquez 2016].

Although 30-day mortality was not significantly different between ICM and non-ICM groups, there were some differences in complication rates. The ICM cohort was found to have a significantly higher incidence of pulmonary complications when compared with the non-ICM group. This can be explained in part by the fact that patients in the ICM group had a lower forced expiratory volume in one second (FEV1) and worse functional independence when compared with the non-ICM group (Table 1). Frailty has been shown to be a risk for postoperative complications including mortality, thus posing another explanation as to why patients in our cohort with ICM had higher long-term mortality [Lee 2010]. Furthermore, although not statistically significant, there was a $6 \%$ higher proportion of smokers in the ICM group. Smoking is a risk factor for the development of ICM as well as CVD in general, as well as myriad other complications. Previous studies have shown that with respect to CABG, smoking increases the risk of pulmonary complications and death [Saxena 2013; Sørensen 2012]. When comparing outcomes between ONCAB and OPCAB in patients with ICM, there was a strong trend toward improved 30-day survival in the OPCAB group, although not statistically significant $(P=.07)$. The failure to reach significance likely is due to low power in our secondary analysis with only 201 patients. These results are consistent with the findings by Puskas et al, who found in a retrospective analysis that high risk patients have a greater mortality benefit from OPCAB as compared to ONCAB when CPB was not used [Dewey 2004]. On the contrary, our OPCAB outcomes in patients with ICM are not congruent with the outcomes of the ROOBY trial, which showed that OPCAB had worse survival and more adverse events at 5 -year follow up [Shroyer 2017]. A major difference, however, was that in our study, patients were not randomized to off-pump versus on-pump prior to the operation, so selection bias likely was present. Furthermore, a criticism of the ROOBY trial was that their surgeons were inexperienced in the OPCAB approach, whereas the surgeons in our study are facile with OPCAB.

What was statistically significant in our secondary analysis was that there were fewer pulmonary complications and fewer returns to OR for bleeding with OPCAB. The benefits of OPCAB that have been explained in literature previously in civilian patients can likely be applied to our cohort as well. A recent meta-analysis by Sepehripour et al highlighted the reasons why OPCAB offers end organ protection. There is less manipulation of the aorta, thus reducing the risk of cerebral embolization during surgery. There also is an inherent inflammatory reaction to $\mathrm{CPB}$ itself, which can lead to pulmonary and renal injury [Sepehripour 2013]. Furthermore, the frequent use of anticoagulation in CPB is not a necessity in the off-pump approach, which likely reduces the incidence of reoperation for bleeding in OPCAB [Sepehripour 2014]. These findings provide an explanation for the findings of fewer pulmonary complications and returns to the OR that we saw in our cohort.

We believe that the part of achieving success after revascularization in patients with ICM is the multidisciplinary patient assessment. All veterans with ICM undergo assessment of myocardial viability of left anterior descending artery territory and evaluation of right ventricular function. This 
Table 2. Postoperative outcomes after coronary artery bypass grafting

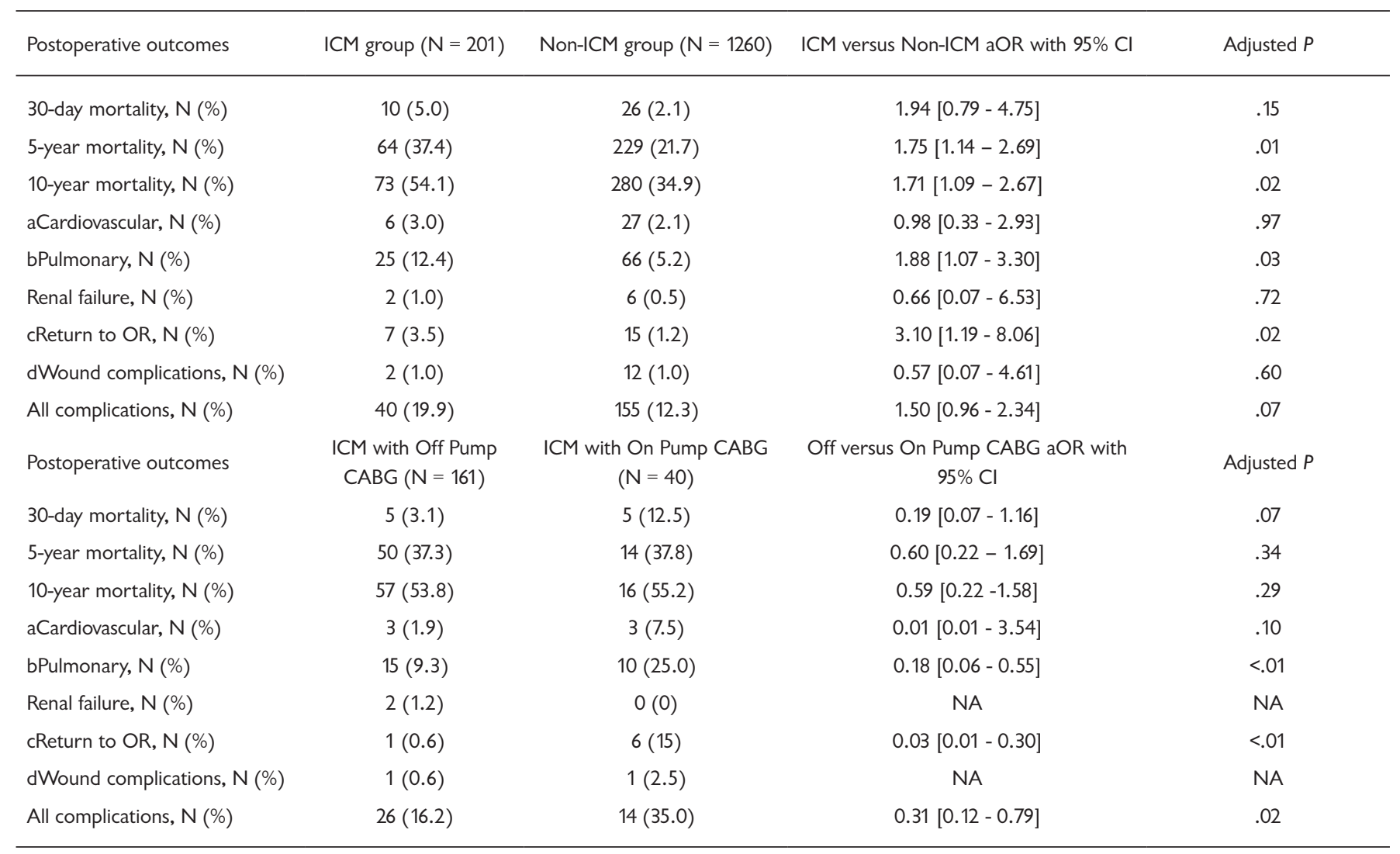

Abbreviations: ICM, ischemic cardiomyopathy; CABG, coronary artery bypass grafting; aOR, adjusted odds ratio; NA, Not applicable due to low N; OR, operating room; aCardiovascular = composite outcome of postoperative myocardial infarction, cardiac arrest, and stroke; bPulmonary = composite outcome of failure to wean from ventilator $>48$ hours, reintubation, tracheostomy, and pneumonia; cReturn to OR = postoperative bleeding requiring return to the operating room. dWound complications = includes superficial and deep wound infections

process has allowed us to identify patients who will benefit the most following revascularization. The same deliberation in patient selection is applied with regard to performing ONCAB versus OPCAB. Ultimately, the decision of whether to do CABG with or without CPB should be personalized to each individual patient, and this study should give surgeons further comfort in knowing that short-term outcomes are improved in the off-pump approach, when applied to the appropriate patient population.

There are several limitations to this study. The inherent weaknesses of a retrospective analysis are present. In addition, the outcomes largely belong to a single operator in the patient population involved in this analysis, which may limit the generalizability of the study. It is important to note that the primary operator for our series is very experienced in the off-pump approach. OPCAB technically is more challenging, and prior literature - including the CORONARY and GOPCABE trials - have shown that more experienced operators achieve better outcomes, including less bleeding, less renal dysfunction, and shorter hospital stay [Diegeler 2019; Lamy 2016; Sellke 2005]. Furthermore, our patient population was almost entirely male. Females actually have been shown to have worse short-term outcomes when undergoing CABG, and inclusion of more females might have altered our results [Saxena 2012].

In conclusion, veterans with ICM undergoing CABG demonstrated comparable short-term survival compared to non-ICM veterans. However, the long-term survival in ICM cohort still is inferior to patients without ICM. Veterans had comparable outcomes to the civilian population, despite a higher risk profile. There is a strong trend of improved shortterm survival and less short-term complications in patients with ICM undergoing OPCAB compared with ONCAB, and multidisciplinary assessment should be employed when deciding which approach to use.

\section{REFERENCES}

Assari S. 2014. Veterans and risk of heart disease in the United States: A cohort with 20 years of follow up. Int J Prev Med 5(6):703-709.

Benjamin EJ, Virani SS, Callaway CW, et al. 2018. Heart Disease and Stroke Statistics-2018 Update: A Report From the American Heart Association. Circulation 137(12):e67-e492. 
Dewey TM, Herbert MA, Prince SL, et al. 2004. Avoidance of Cardiopulmonary Bypass Improves Early Survival in Multivessel Coronary Artery Bypass Patients with Poor Ventricular Function. Heart Surg Forum 7(1):45-50.

Diegeler A, Börgermann J, Kappert U, et al. 2019. Five-Year Outcome after Off-Pump or On-Pump Coronary Artery Bypass Grafting in Elderly Patients. Circulation 139(16):1865-1871.

Groeneveld PW, Medvedeva EL, Walker L, Segal AG, Richardson DM, Epstein AJ. 2018. Outcomes of Care for Ischemic Heart Disease and Chronic Heart Failure in the Veterans Health Administration. JAMA Cardiology 3(7):563.

Lamy A, Devereaux PJ, Prabhakaran D, et al. 2016 Five-Year Outcomes after Off-Pump or On-Pump Coronary-Artery Bypass Grafting. New England Journal of Medicine 375(24):2359-2368.

Lee DH, Buth KJ, Martin B, Yip AM, Hirsch GM. 2010. Frail patients are at increased risk for mortality and prolonged institutional care after cardiac surgery. Circulation 121(8):973-978.

Matula SR, Trivedi AN, Miake-Lye I, Glassman PA, Shekelle P, Asch S. 2010. Comparisons of quality of surgical care between the US department of veterans affairs and the private sector. J Am Coll Surg 211(6):823-832.

Puskas JD, Thourani VH, Kilgo P, et al. 2009. Off-pump coronary artery bypass disproportionately benefits high-risk patients. Ann Thorac Surg 88(4):1142-1147.

Rezapour A, Bagheri Faradonbeh S, Alipour V, Yusefvand M. 2018. Effectiveness of revascularization interventions compared with medical therapy in patients with ischemic cardiomyopathy: A systematic review protocol. Medicine (Baltimore) 97(10):e9958.

Rosenthal GE, Vaughan Sarrazin M, Hannan EL. 2003. In-hospital mortality following coronary artery bypass graft surgery in veterans health administration and private sector hospitals. Med Care 41(4):522-535.

Saxena A, Dinh D, Smith JA, Shardey G, Reid CM, Newcomb AE. 2012. Sex differences in outcomes following isolated coronary artery bypass graft surgery in Australian patients: Analysis of the Australasian society of cardiac and thoracic surgeons cardiac surgery database. Eur J Cardiothorac Surg 41(4):755-762.

Saxena A, Shan L, Reid C, et al. 2013. Impact of smoking status on early and late outcomes after isolated coronary artery bypass graft surgery. J Cardiol 61(5):336-341.

Sellke FW, DiMaio JM, Caplan LR, et al. 2005. Comparing on-pump and off-pump coronary artery bypass grafting: Numerous studies but few conclusions: A scientific statement from the American heart association council on cardiovascular surgery and anesthesia in collaboration with the interdisciplinary working group on quality of care and outcomes research. Circulation 111(21):2858-2864.

Sepehripour AH, Harling L, Ashrafian H, Casula R, Athanasiou T. 2014. Does off-pump coronary revascularization confer superior organ protection in re-operative coronary artery surgery? A meta-analysis of observational studies. J Cardiothrac Surg 9:115.

Sepehripour AH, Saso S, Harling L, Punjabi P, Ashrafian H, Athanasiou T. 2013. Does off-pump coronary revascularization reduce mortality in re-operative coronary artery surgery? A meta-analysis of observational studies. Perfusion 28(4):340-349.

Shroyer AL, Hattler B, Wagner TH, et al. 2017. Five-Year Outcomes after On-Pump and Off-Pump Coronary-Artery Bypass. New England Journal of Medicine 377(7):623-632.

Sørensen LT. 2012. Wound healing and infection in surgery: The pathophysiological impact of smoking, smoking cessation, and nicotine replacement therapy: A systematic review. Ann Surg 255(6):1069-1079.

Trachiotis GD, Weintraub WS, Johnston TS, Jones EL, Guyton RA, Craver JM. 1998. Coronary Artery Bypass Grafting in Patients with Advanced Left Ventricular Dysfunction. Ann Thorac Surg 66:1632-9.

Velazquez EJ, Lee KL, Jones RH, et al. 2016. Coronary-Artery Bypass Surgery in Patients with Ischemic Cardiomyopathy. New England Journal of Medicine 374(16):1511-1520. 